ISSN-i 1390-7042 | ISSN-e 2602-8484

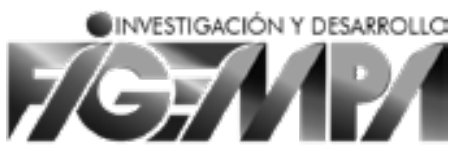

\title{
FACTORES QUE INCIDEN EN LA PROBABILIDAD DE OCURRENCIA DE INCENDIOS FORESTALES EN ECUADOR
}

\section{Factors that influence the probability of occurrence of forest fires in Ecuador}

Carla Balcázar Gallegos

Universidad Técnica Particular de Loja

cibalcazar@utpl.edu.ec

ORCID: https://orcid.org/0000-0002-5646-0263
Fabián Reyes Bueno

Universidad Técnica Particular de Loja

frreyes@utpl.edu.ec

ORCID: https://orcid.org/0000-0002-5646-0263
Recibido: septiembre de 2020

Aprobado: mayo de 2021

ARTÍCULO ORIGINAL

DOI: $10.29166 /$ revfig.v11i1.2634
PALABRAS CLAVE Incendios forestales, gestión del fuego, análisis espacial, MaXent.

KEYWORDS Forest fires, fire management, spatial analysis, MaXent.

\section{RESUMEN}

Los incendios forestales son un problema ambiental y socioeconómico, la frecuencia y área afectada por estos eventos se atribuyen al cambio climático y las actividades antropogénicas que se desarrollan en el territorio. Como parte de los programas de prevención, es necesario identificar y limitar las áreas susceptibles a incendios forestales para la priorización de recursos por parte de los entes encargados. Los sistemas de información geográfica y MaXent (modelos de entropía máxima) son usados para este fin. Los datos de ocurrencia de incendios fueron obtenidos de la plataforma Active fire data. Como variables independientes se obtuvo información del clima del WorldClim, y se generaron variables de accesibilidad para la zona de estudio, en el periodo 2012-2018. En este modelo se identificó que la variable distancia a zonas antrópicas es la de mayor importancia y con ello el predictor más susceptible a un incendio forestal en el cantón Loja, seguida de las variables climáticas: temperatura mínima en el mes más frío y la precipitación en el trimestre más seco; estas tres variables coinciden dentro de las características que se señalan en los pocos estudios encontrados y realizados por entidades públicas a nivel nacional. Se analizó de forma superficial el tiempo de llegada del cuerpo de bomberos a las áreas identificadas como susceptibles, obteniéndose que casi el 99,96\% de éstas se encuentran fuera del tiempo de atención óptimo (5 min) y un 32,94\% están dentro del radio de acción de $7 \mathrm{~km}$, lo cual tiene injerencia negativa en el control de un incendio forestal.

\section{ABSTRACT}

Forest fires are an environmental and socioeconomic problem; the frequency and area affected by these events are attributed to climate change and anthropogenic activities in the territory. As part of the prevention programs, it is necessary to identify and limit the areas susceptible to forest fires for the prioritization of resources by the responsible entities. Geographic information systems and MaXent (maximum entropy models) are used for this purpose. Fire occurrence data were obtained from the Active fire data platform. As independent variables, climate information was obtained from WorldClim, and accessibility variables were generated for the study area, in the period 2012-2018 In this model it was identified that the variable distance to anthropic zones is the most important and thus the most susceptible predictor to a forest fire in 
Loja canton, followed by the climatic variables minimum temperature in the coldest month and precipitation in the driest quarter; these three variables coincide within the characteristics that are indicated in the few studies found and by public entities at the national level. A superficial analysis was made of the arrival time of the Fire Department to the areas identified as susceptible, showing that almost $99.96 \%$ of these areas are outside the optimal response time ( $5 \mathrm{~min}$ ) and $32.94 \%$ are within the $7 \mathrm{~km}$ radius of action, which has a negative impact on the control of a forest fire.

\section{INTRODUCCIÓN}

En los últimos años los incendios representan una amenaza para los ecosistemas forestales, sean éstos originados por factores naturales o antropogénicos; a su vez, el aumento en la frecuencia y la gravedad en estos eventos se ven potenciados por el cambio climático, el aumento de la temperatura y la sequedad, los cambios en el uso de la tierra y las intensas actividades antropogénicas (Ghomi, Farahi, Baniasadi y Masoumpoor, 1937; Crimmins, 2006; Running, 2006; Chen et al., 2012; Hantson, Pueyo y Chuvieco, 2014; Eskandari y Chuvieco, 2015), agravando problemas socioambientales del territorio, por lo cual es necesario que los entes encargados de prevenir estos eventos, conservar ecosistemas y gobiernos locales que trabajan en la ordenación del territorio, adopten herramientas que permitan cumplir con dos acciones: a) evaluar la susceptibilidad al fuego, predecir la posible ocurrencia de incendios en el futuro e identificar áreas de alto riesgo; $\mathrm{y} b$ ) tomar decisiones con respecto a la financiación, desarrollo y despliegue de recursos de extinción y prevención de incendios; sin embargo, el proceso natural que implica la ignición y propagación de incendios forestales tiene una naturaleza compleja y no lineal debido a la incidencia de factores como el clima, topografía, cobertura vegetal, actividad antrópica, etc.; lo que lo hace un evento complejo de predecir (Jaiswal, Mukherjee, Raju y Saxena, 2002; Pourghasemi, 2016; Tien Bui et al., 2017; Ngoc Thach et al., 2018).

A nivel mundial, plataformas como Active Fire Data, World View y Global Forest Watch, permiten identificar las áreas en peligro de incendio a través de anomalías térmicas detectadas por el sensor MoDIs (Justice et al., 2002; Renard, et al., 2012). En Ecuador contamos con un modelo nacional de susceptibilidad a incendios forestales construido a partir del análisis físico del fenómeno y está orientado a definir el origen, comportamiento en la ignición, considerando criterios heurísticos y la ponderación de variables; la propuesta metodológica de este modelo de susceptibilidad a incendios forestales se adapta a escala nacional y regional según la Secre- taría Nacional de Gestión de Riesgos y Emergencias (SNGRE, 2017).

Para identificar la susceptibilidad a incendios forestales a diferentes escalas, ecosistemas y localidades en diferentes partes del mundo, se han utilizado varios métodos y algoritmos por medio de los sistemas de información geográfica y técnicas de teledetección, entre las que destacan en los últimos tiempos las técnicas de minería de datos, debido a que permiten analizar problemas complejos de múltiples variables y construir modelos predictivos a partir de grandes conjuntos de datos. Entre las técnicas que se usan, están el modelo lineal generalizado (GLM) (Bar Massada et al., 2012); los árboles de clasificación y regresión (CART) (Amatulli et al., 2006); la regresión múltiple logística (LMR), bosque aleatorio (RF) es un desarrollo de métodos de clasificación y árbol de regresión (Oliveira et al., 2012); redes neuronales artificiales (ANA) (Bisquert et al., 2012); árboles de regresión impulsado (BRT) es una combinación de técnicas estadísticas y de aprendizaje automático y una extensión de CART; máquina de vectores de soporte ( $\mathrm{svm}$ ) (Hong et al., 2018); red neuronal perceptrónica multicapa (MLP) (Ngoc Thach et al., 2018); regresión logística del núcleo (KLR) (Zhang, Wang y Liu, 2019); árboles de decisión impulsados por gradiente (Sachdeva, Bhatia y Verma, 2018); modelos de optimización de enjambre de partículas (Tien Bui et al., 2017); MaXent que, en sí, es un modelo de aprendizaje automático disponible como software e implementado en PC (Phillips, Anderson y Schapire, 2006), el principio de este último recurso consiste en estimar la distribución de probabilidad de la entropía máxima (la más extendida o más cercana al uniforme) basado en dos conjuntos de datos: a) un conjunto de variables (tipo ráster) que en el caso de los incendios forestales se relacionan con el «triángulo del fuego»; b) registros georreferenciados de "presencia» de incendios, a partir de los datos mencionados se obtiene un mapa (tipo ASCCI) que muestra la gradiente de probabilidad de ocurrencia de incendio por pixel, lo que resulta en un mapa de susceptibilidad, en donde los valores cercanos a 100 representan los pixeles que cumplen con las carac- 
terísticas para que se origine un incendio forestal y los valores cercanos a cero no cumplen con estas características (Phillips, Anderson y Schapire, 2006; Renard et al., 2012; Ibarra-Montoya y Huerta-Martínez, 2016).

Como se puede percibir, el modelo a obtenerse en Maxent es confiable y fácil de conceptualizar (Elith et al., 2006; Correia, 2019); sumado a la sencillez de su interfaz gráfica y al uso libre del software lo hace un programa altamente utilizado en diferentes estudios afines a la problemática en cuestión (Bar Massada et al., 2012; Ibarra-Montoya y Huerta-Martínez, 2016; Garrido et al., 2018).

Con estos antecedentes, el presente estudio pretende dar datos empíricos para una planificación eficiente en prevención y mitigación de los incendios forestales en Ecuador, a través de un estudio de caso en el cantón Loja; identificando áreas susceptibles a incendios forestales a partir de un modelo construido en Maxent, con datos de presencia de incendios forestales obtenidos del sensor modis y combinaciones de variables predictoras de estos eventos, pretendiendo que el modelo también pueda ser replicado en otras localidades. A su vez, como parte del control del fuego se identifica el tiempo de llegada del Cuerpo de Bomberos.

\section{MATERIALES Y MÉTODOS}

\section{ÁREA DE ESTUdIO}

El cantón Loja se encuentra al sur del Ecuador (ver Figura 1), forma parte de la jurisdicción política de la provincia que tiene el mismo nombre y posee una superficie aproximada de $1895,53 \mathrm{~km}^{2}$. El clima es templado-ecuatorial, subhúmedo, con una temperatura promedio de $16-24^{\circ} \mathrm{C}$ y un rango altitudinal de 1200 3800 m s. n. m. (Gobierno Provincial de Loja, 2016).

La época de mayor incidencia de incendios forestales se presenta durante los meses de julio a diciembre (Galindo, Sarango y Villarroel, 2012). Estos incendios suelen ocurrir, sobre todo, por la quema descontrolada de matorrales y potreros para un nuevo periodo de cultivo, según el Plan de Ordenamiento Territorial del Gobierno Autónomo Descentralizado del Municipio de Loja (GAD Municipio de Loja, 2014).

\section{METODOLOGíA}

En esta sección primero se describe el desarrollo del modelo de susceptibilidad a través del preprocesamiento de datos y el ajuste de MaXent para la obtención del modelo final y clasificación de probabilidad.

\section{DATOS DE LA PRESENCIA DE INCENDIO FORESTALES}

De la plataforma Active fire data (https://firms.modaps.eosdis.nasa.gov/active_fire/\#firms-shapefile), se descargaron los datos georreferenciados de incendios forestales del sensor MODIs (espectro radiómetro de imágenes de media resolución), satélite que fue el primero en monitorear los incendios a través de la detección de anomalías térmicas por pixel (Justice et al., 2002; Renard et al., 2012). La información relacionada con cada registro y relevante para el presente estudio incluyó la ubicación (latitud y longitud) y fecha. La confiabilidad y precisión de MODIs ha sido comprobada en estudios como el de Huesca, González-Alonso y Cuevas (2009); Merino-de-Miguel, Gonza y Huesca (2011); Renard et al. (2012). Si bien el sensor presenta conflictos en la obtención de valores de reflectividad de superficie por la presencia de nubes y puede confundir las sombras de las nubes como área quemada, los datos de éste nos aproximan a la realidad de la problemática (Jin y Sader, 2005; Giglio et al., 2018; Roteta et al., 2019). Para el periodo 2012-2018, se seleccionó aquellos datos correspondientes al área de estudio y se filtró para escoger los que se encontraban en áreas de vegetación nativa y plantación forestal.

\section{PREDICTORES MEDIOAMBIENTALES}

Consideramos diferentes conjuntos y fuentes predictores ambientales de ocurrencia de incendios, los mismos se resumen en la tabla 1.

\section{Predictores antrópicos}

Teniendo en cuenta que la variable antrópicos es una fuerte predictora de incendios forestales según (Chuvieco et al., 2007; Vilar del Hoyo et al., 2007) y, según la Secretaría Nacional de Gestión de Riesgos el 99\% de los incendios forestales en el Ecuador se dan por la intervención del hombre (Ocles, 2008). Se crearon a partir de las capas tipo.$s h p^{*}$ de vías, ríos, pendiente, cobertura vegetal y centros económicos; tres capas de accesibilidad, es decir, capas que nos indiquen el tiempo de desplazamiento desde cada celda hacia el centro económico, vías, zonas antrópicas y estaciones de bomberos, esta última capa no fue parte del modelo. Las capas obtenidas fueron de tipo ráster y con tamaño de celda de $100 \mathrm{~m}^{2}$.

\section{Predictores ambientales}

Diecinueve capas bioclimáticas con resolución espacial de $1 \mathrm{~km} 2$ se descargaron de la página World Clim (versión 1.4) (Fick y Hijmans, 2017), estas fueron cortadas para el área de estudio y reproyectadas al sistema wgs84/utm-17s (ver Tabla 1).

Los datos tipo ráster se los transformó a archivos ASCII (archivo compatible con MaXent) y se verificó 


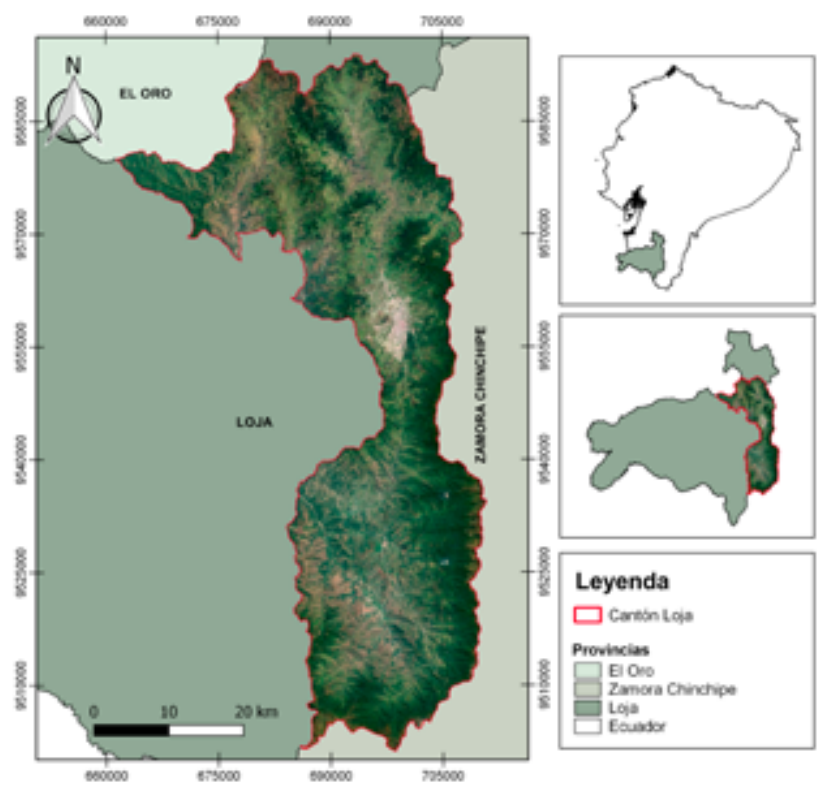

Figura 1. Mapa de ubicación geográfica del cantón Loja, provincia de Loja-Ecuador.

Tabla 1. Las variables predictoras de incendios forestales y utilizadas en el primer modelo.

\begin{tabular}{|c|c|c|c|c|c|c|}
\hline N.o & Variable & & $\begin{array}{l}\text { Resolución } \\
\text { /Escala }\end{array}$ & Unidad & $\begin{array}{l}\text { Formato origi- } \\
\text { nal de datos }\end{array}$ & Fuente \\
\hline 1 & $\begin{array}{l}\text { Distancia a zonas } \\
\text { antrópicas }\end{array}$ & Elevación/pendiente & $30 \mathrm{~m}$ & Grados & Ráster & $\begin{array}{l}\text { OpenTopo- } \\
\text { graphy }\end{array}$ \\
\hline 2 & $\begin{array}{l}\text { Distancia } \\
\text { a centros económicos }\end{array}$ & Cobertura vegetal & $1: 50000$ & M & Shape/ Vector & $\begin{array}{l}\text { Instituto } \\
\text { Geográfico } \\
\text { Militar }\end{array}$ \\
\hline \multirow[t]{2}{*}{3} & Distancia a vías & Ríos & $1: 50000$ & M & Shape/ Vector & $\begin{array}{l}\text { Instituto } \\
\text { Geográfico } \\
\text { Militar }\end{array}$ \\
\hline & & Vías & $1: 5000 \mathrm{o}$ & M & Shape/ Vector & $\begin{array}{l}\text { Instituto } \\
\text { Geográfico } \\
\text { Militar }\end{array}$ \\
\hline 4 & \multicolumn{2}{|c|}{ BIO1 Temperatura media anual } & $1 \mathrm{~km}$ & ${ }^{\circ} \mathrm{C}$ & Ráster & WorldClim \\
\hline 5 & \multicolumn{2}{|c|}{$\begin{array}{l}\text { BIO2 Intervalo diurno medio (promedio mensual } \\
\text { (temperatura máxima - temperatura mínima) }\end{array}$} & $1 \mathrm{~km}$ & ${ }^{\circ} \mathrm{C}$ & Ráster & WorldClim \\
\hline 6 & \multicolumn{2}{|c|}{ BIO3 Isotermia (BIO2 / BIO7) $\left({ }^{\star} 100\right)$} & $1 \mathrm{~km}$ & ${ }^{\circ} \mathrm{C}$ & Ráster & WorldClim \\
\hline 7 & \multicolumn{2}{|c|}{$\begin{array}{l}\text { BIO4 Temperatura estacional (desviación estándar } \\
\text { * 100) }\end{array}$} & $1 \mathrm{~km}$ & ${ }^{\circ} \mathrm{C}$ & Ráster & WorldClim \\
\hline 8 & \multicolumn{2}{|c|}{ BIO5 Temperatura máxima del mes más cálido } & $1 \mathrm{~km}$ & ${ }^{\circ} \mathrm{C}$ & Ráster & WorldClim \\
\hline 9 & \multicolumn{2}{|c|}{ BIO6 Temperatura mínima del mes más frío } & $1 \mathrm{~km}$ & ${ }^{\circ} \mathrm{C}$ & Ráster & WorldClim \\
\hline 10 & \multicolumn{2}{|c|}{ BIO7 Rango anual de temperatura (BIO5 - BIO6) } & $1 \mathrm{~km}$ & ${ }^{\circ} \mathrm{C}$ & Ráster & WorldClim \\
\hline 11 & \multicolumn{2}{|c|}{$\begin{array}{l}\text { BIO8 Temperatura media de trimestre más húme- } \\
\text { do }\end{array}$} & $1 \mathrm{~km}$ & ${ }^{\circ} \mathrm{C}$ & Ráster & WorldClim \\
\hline
\end{tabular}




\begin{tabular}{llllll}
12 & BIO9 Temperatura media del trimestre más seco & $1 \mathrm{~km}$ & ${ }^{\circ} \mathrm{C}$ & Ráster & WorldClim \\
13 & BIO10 Temperatura media del trimestre más cálido & $1 \mathrm{~km}$ & ${ }^{\circ} \mathrm{C}$ & Ráster & WorldClim \\
14 & BIo11 Temperatura media del trimestre más frío & $1 \mathrm{~km}$ & ${ }^{\circ} \mathrm{C}$ & Ráster & WorldClim \\
15 & BIO12 Precipitación anual & $1 \mathrm{~km}$ & $\mathrm{~mm}$ & Ráster & WorldClim \\
16 & BIo13 Precipitación del mes más húmedo & $1 \mathrm{~km}$ & $\mathrm{~mm}$ & Ráster & WorldClim \\
17 & BIO14 Precipitación del mes más seco & $1 \mathrm{~km}$ & $\mathrm{~mm}$ & Ráster & WorldClim \\
18 & $\begin{array}{l}\text { Bio15 Estacionalidad de la precipitación (coefi- } \\
\text { ciente de variación) }\end{array}$ & $1 \mathrm{~km}$ & $\mathrm{~mm}$ & Ráster & WorldClim \\
19 & BIO16 Precipitación del trimestre más húmedo & $1 \mathrm{~km}$ & $\mathrm{~mm}$ & Ráster & WorldClim \\
20 & BIO17 Precipitación del trimestre más seco & $1 \mathrm{~km}$ & $\mathrm{~mm}$ & Ráster & WorldClim \\
21 & Bio18 Precipitación del trimestre más cálido & $1 \mathrm{~km}$ & $\mathrm{~mm}$ & Ráster & WorldClim \\
22 & BIo19 Precipitación del trimestre más frío & $1 \mathrm{~km}$ & $\mathrm{~mm}$ & Ráster & WorldClim \\
\hline
\end{tabular}

MaXent: modelo de incendios

que estén en una misma resolución y extensión espacial. Antes de obtener el modelo final se realizó un modelo previo, para identificar las variables de mayor importancia. En el primer modelo se utilizaron las diecinueve capas bioclimáticas y tres capas de accesibilidad (Tabla 1), estas capas se ajustaron a un tamaño en común de $100 \mathrm{~m}^{2}$.

En el segundo modelo se realizó un análisis de correlación (Pearson) entre las veintidós capas, eliminándose aquellas variables que resultaron tener una fuerte correlación $(>0,8)$ con las variables que de acuerdo con el primer modelo contribuían más. Finalmente, fueron seleccionadas doce variables para generar el modelo definitivo (ver Figura 2). En ambos modelos, el 25\% de los 170 datos obtenidos fueron utilizados para la evaluación del modelo, y se realizó un test de Jackknife para identificar las variables que tienen mayor peso dentro del modelo a obtenerse (Phillips, Anderson y Schapire, 2006).

Para validar el modelo final, se evaluó su rendimiento en función del Roc (Receiver Operating Characteristic), comúnmente utilizado para evaluar modelos de distribución y que proporciona un área bajo la curva como una medida general del modelo (Fielding y Bell, 1997; Phillips, Anderson y Schapire, 2006).

\section{Mapeo de susceptibilidad de incendios forestales y tiempo de accesibilidad}

El modelo resultante fue un modelo logístico, que fue reclasificado en 3 rangos de probabilidad (ver Tabla 2) elegidos a partir del criterio del autor, esto permitió obtener un archivo shape $\left({ }^{*}\right.$.shp) y eliminar los datos de una probabilidad baja.

También se creó una capa de accesibilidad a esta- ciones de bomberos y en esta se definió intervalos de tiempo de 0-5, 5-30, 30-60 y de más de $60 \mathrm{~min}$. En la capa de cobertura vegetal se eliminó aquellos datos que correspondían a un uso antrópico, dejándose solo los datos de vegetación natural y plantaciones forestales. Las tres capas provenientes del proceso anterior fueron unidas, lo que permitió identificar aquellas zonas de vegetación natural o plantaciones forestales con probabilidad media o alta de incendio y tiempo de accesibilidad a esos sitios.

\section{RESULTADOS}

\section{MODELO PREDICTIVO MAXENT}

La robustez del modelo se ve respaldada por los estudios de Welsh et al. (1996); Peterson et al. (2000); Stockwell y Peterson (2002), que mencionan que se debe utilizar como mínimo 50 puntos y tomar en cuenta la resolución espacial para tener una buena aproximación de las condiciones del territorio; en el presente estudio se utilizó 170 puntos de presencia y variables con una resolución espacial fina (pixeles de $1 \mathrm{~km}^{2}$ ). Con respecto a la calidad del modelo, según Parolo, Rossi y Ferrarini (2008) y Correia (2019), es buena al arrojarse un área bajo la curva (AUC) de 0,872 para los datos de enteramiento (training data). En la prueba Jackknife las variables que tuvieron mayor ponderación dentro del modelo son: distancia a zonas antrópicas, la temperatura media del trimestre más frío (Bio6) y la precipitación del trimestre más seco (Bio17).

Analizando las curvas de repuesta generadas en el software, las zonas que tienen una susceptibilidad media de que ocurra un incendio forestal son aquellas que se encuentra a una distancia menor a $2700 \mathrm{~m}$ de 
Tabla 2. Clasificación de los valores únicos asignados a la probabilidad de incendios forestales

\begin{tabular}{lll}
\hline Valor de celda & Valor único asignado & Clasificación \\
\hline $0 \mathrm{a}<0,5$ & 0 & Baja \\
$>=0,5 \mathrm{a}<=0,8$ & 1 & Media \\
$>0,8$ & 2 & Alta \\
\hline
\end{tabular}

las zonas antrópicas, siendo éste el predictor más fuerte para la ocurrencia de incendios forestales; con respecto a la variable temperatura mínima en el mes más frío que corresponde al mes de julio y la precipitación en el trimestre más seco que corresponde a los meses de julio, agosto, septiembre, según el Instituto Nacional de Meteorología e Hidrología (INAMHI, 2018). Si existe una temperatura que varía entre 9,2 y $12,5^{\circ} \mathrm{C}$; y una precipitación de $75 \mathrm{~mm}$, existe mayor probabilidad de que ocurra un incendio forestal en dichos meses.

\section{ÁREA SUSCEPTIBLE A INCENDIOS FORESTALES}

Existe un total de 26.812,48 (20,48\%) de las 130.892,35 ha de cobertura natural del cantón, que tienen una probabilidad entre el 50\%-80\% de ocurrencia de incendios forestales. La vegetación arbustiva y herbácea representan el $21,33 \%$ y $51,34 \%$ del área identificada, respectivamente; la cobertura forestal es la menos susceptible a incendios forestales, con un área que representa el 2,93\% de la identificada (ver Figura 3a).

\section{ACCESIBILIDAD EN MINUTOS A LAS ÁREAS SUS- CEPTIBLES A INCENDIOS FORESTALES}

El 32,94\% (8833 ha) del área susceptible a incendios forestales se encuentra dentro del radio de acción de 7 $\mathrm{km}$ de las estaciones de bomberos, de éstas, solo 10,67 ha tienen un tiempo de llegada de 5 min del Cuerpo de Bomberos, tiempo recomendado por la SNGRE (2016) y la National Fire Protection Association (NFPA, 2018). Para identificar la calzada de las vías de acceso a las zonas identificadas como susceptibles a incendios forestales, se realizó una consulta espacial en el software QGIs. Una buena parte de las zonas que tienen un tiempo de llegada mayor a $60 \mathrm{~min}$ tienen acceso por vías de tierra; las que están en un tiempo de 30 a 60 min tienen vías de tierra y lastradas, y las zonas con un tiempo de llegada de 5 a 30 min y de 0 a $5 \mathrm{~min}$, tienen los tres tipos de vías: de tierra, lastrada y de pavimento. En la figura $3 b$ se identifica el área afectada de incendios según el tiempo de llegada del Cuerpo de Bomberos (ver Figura 3b).

\section{DISCUSIóN}

$\mathrm{Al}$ comportarse como un proceso estocástico la ignición por fuego y el entorno variable de nuestra área de estudio, no se puede dar conclusiones generales sobre el proceso de pre-ignición; sin embargo, se identifica que la variable distancia a zonas antrópicas es el predictor que incide en la mayor ocurrencia de incendios forestales. Este resultado concuerda con los estudios realizados por Sturtevant y Cleland (2007); Syphard et al. (2007); Bar Massada et al. (2012); Parisien et al. (2012) en diferentes localidades y que proyectan a las actividades antrópicas y cercanía de las poblaciones como la principal causa de incendios forestales; así mismo concuerda de forma local, con lo descrito en el Plan de Ordenamiento Territorial del cantón Loja (GAD Municipio de Loja, 2014). Adicionalmente, las variables climáticas que son fuertes predictoras de incendios forestales en el cantón Loja son la temperatura media del trimestre más frío (Bio6) y la precipitación del trimestre más seco (Bio17), predictores que suelen ocurrir entre los meses de julio y septiembre.

Las variables antropogénicas a menudo se obtienen fácilmente y pueden claramente relacionarse con la distancia o densidad de los incendios, no se puede decir lo mismo de los datos climáticos, los cuales a menudo no tienen la misma resolución y calidad. Esto limita la georreferenciación de áreas problemáticas, pero ayudan estimar la probabilidad de incendio cuando no contamos con datos climáticos multitemporales y cuando hemos limitado los datos de incendios a áreas forestales. Hay que mencionar que el área de estudio, por sus condiciones climáticas y estructura de la vegetación (poca combustibilidad), no presenta incendios como un proceso natural y, por ende, la variable climática no está relacionada directamente en el origen de estos eventos (Arpaci et al., 2014; Merino-de-Miguel, Gonza y Huesca, 2011), pero sí influye en el desarrollo de las actividades antrópicas, de forma especial en la agricultura. Justamente en los meses mencionados es en donde se da la preparación de terreno previa a la siembra agrícola, y por ende se puede apreciar que existe una mayor frecuencia de incendios forestales (Galindo, Sarango y Villarroel, 2012; GAD Municipio de Loja, 2014; Soto, 2017).

Ante la falta de estudios y para validar el modelo, se comparó las áreas (parroquias) identificadas como susceptibles a incendios forestales con datos de fre- 
cuencia de incendios proporcionados por la SNGRE, comprobándose que las áreas identificadas coinciden, y que el modelo puede ser útil para la limitación de áreas susceptibles a incendios forestales y posterior uso eficiente de recursos en las campañas de prevención y mitigación de incendios forestales por parte de las entidades involucradas (Armenteras, González y Franco, 2009; Rodríguez Montellano, 2012; Díaz-Hormazábal y González, 2016). A nivel nacional, el 99\% de los incendios forestales se atribuye a las actividades antrópicas, pero esto no está respaldado con datos estadísticos, por lo cual este estudio también puede contribuir a esa discusión, y evidenciar que las variables climáticas juegan un papel importante en la distribución espacial y origen de los incendios forestales y dar paso a un estudio más profundo sobre su relación multitemporal con los incendios y actividades económicas

Como punto aparte, dentro del control de los incendios forestales es necesario identificar el tiempo de atención del Cuerpo de Bomberos de Loja (CBL). Según la SNGRE (2016) y NFPA (2018) el tiempo óptimo de atención del cuerpo de bomberos a cualquier emergencia, incluyendo incendios forestales es de 5 min, pero en estudios en diferentes partes del mundo se estima un tiempo óptimo entre 4 y 6 min (Liu, Huang y Chandramouli, 2006; Zhang y Jiang, 2012; Savsar, 2014; Satchet et al., 2018). Dicho esto, el 99,96\% de área susceptible a incendios forestales identificada en el presente estudio está fuera del tiempo de atención óptimo establecido por la entidad nacional (SNGRE) y mundial (NFPA), sin embargo, esto no implica una negligencia por parte del CBL ya que este ente basa su ubicación en la concentración poblacional y cobertura territorial, además, que el tiempo de atención se ve afectado por la existencia de barreras físicas, geográficas y la topografía (Aponte, 2016; Díaz, 2018). La importancia de tomar en cuenta el tiempo de atención dentro de los programas de prevención, es que una vez identificadas las áreas susceptibles se podrá implementar brigadas comunitarias (primera respuesta) en sectores problemáticos, las mismas que pueden ayudar en la prevención y control del fuego, y promover prácticas seguras de quema en la agricultura (Food and Agriculture Organization of the United Nations, 2000).

El estudio representa el primer modelo predictivo de susceptibilidad a incendios forestales realizado en el cantón Loja, utilizando un algoritmo de aprendizaje automático como lo es el software MaXent, cuyo uso ha sido puesto en discusión en estudios como los de Phillips, Anderson y Schapire (2006); Chen et al. (2015). La confianza en este software es validada por la predicción que realiza en función de datos de presencia de los eventos y la relación que tienen éstos con las variables del entorno.

\section{CONCLUSIONES Y RECOMENDACIONES}

Los resultados de este estudio nos indican con datos empíricos, que la vegetación nativa y forestal que se encuentre a una distancia no mayor de $2700 \mathrm{~m}$ a zonas en donde se desarrollan las actividades antrópicas, son aquellas que son más susceptibles a la ocurrencia de un incendio forestal. Así mismo, podemos comprobar, y de acuerdo a la variables utilizadas, que la temporada de incendios forestales en el cantón Loja, abarca los meses de julio, agosto y septiembre.

A pesar de que un incendio forestal se comporta como un proceso estocástico, el uso del software MaXent es una herramienta útil, de fácil manejo y conceptualización del modelo a obtenerse En cuanto a incendios forestales, lo que lo hace atractivo para que entidades ajenas a la academia, puedan utilizarlo y ejecutar a partir de los resultados, programas de mitigación, prevención y control. Recalcando que el rol de la academia siempre será importante para el desarrollo de investigación con respecto al proceso de preignición, ignición, afectación y recuperación de una zona quemada.

Levantar información con respecto a la accesibilidad de los servicios de emergencia daría información valiosa con respecto a su ubicación y eficiencia de atención a zonas problemáticas o alejadas, siendo este un punto que se debe empezar a investigar. 

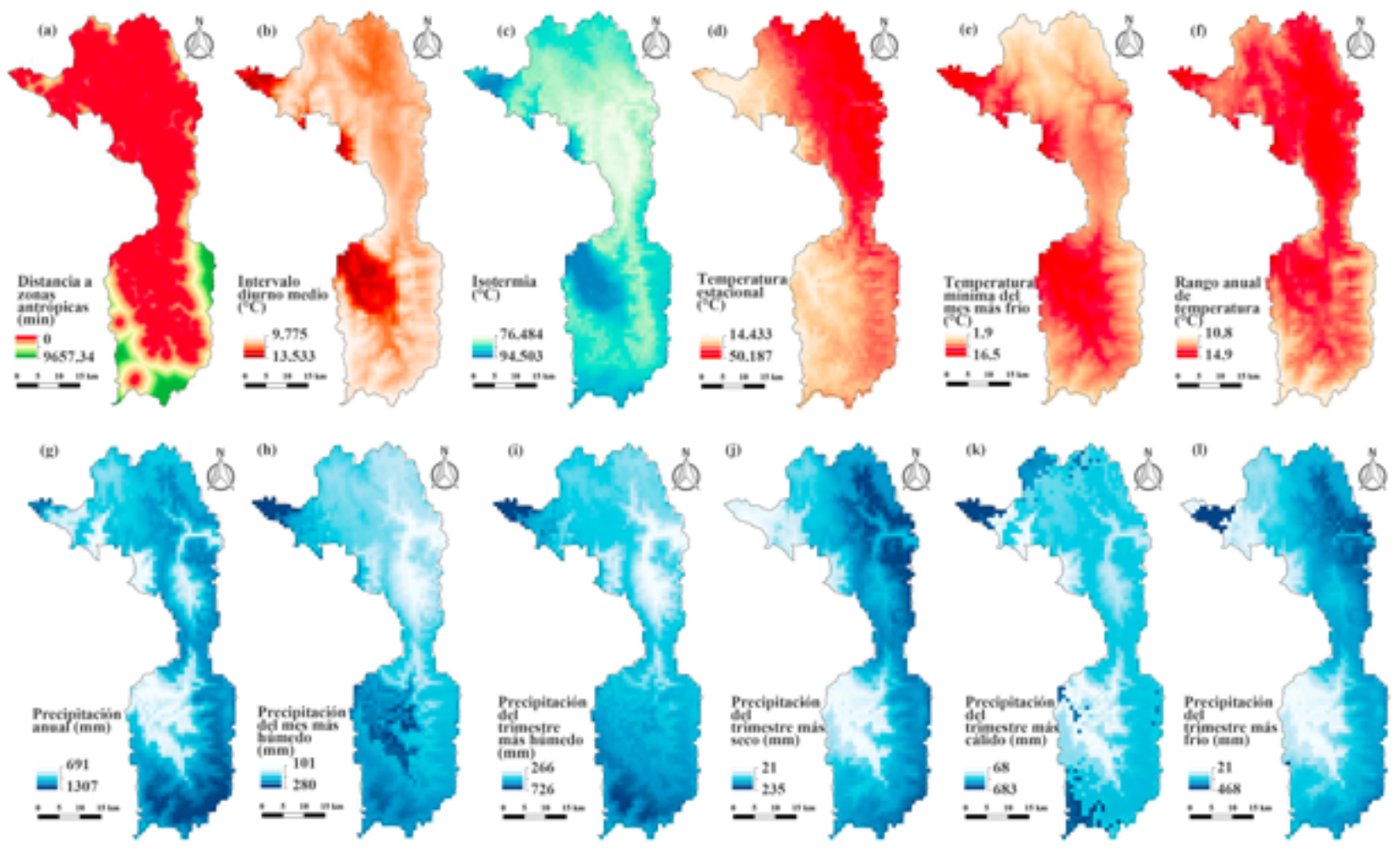

Figura 2. Variables utilizadas en el modelo final.
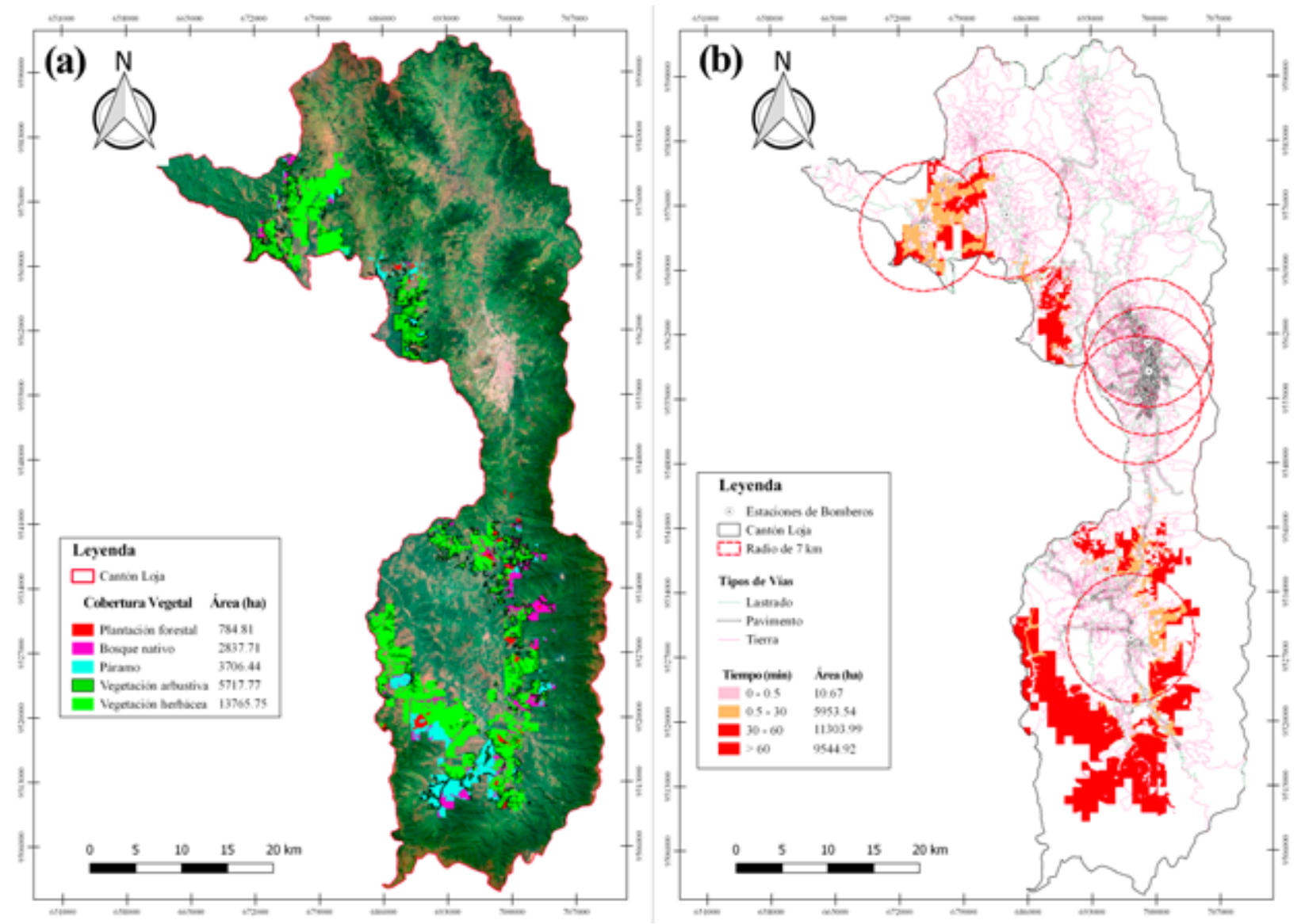

Figura 3a. Cobertura vegetal y forestal susceptible a incendios forestales.

Figura 3b. Tiempo de accesibilidad del Cuerpo de Bomberos hacia las áreas de mayor probabilidad a incendios forestales. 


\section{REFERENCIAS}

Amatulli, G., Rodrigues, M., Trombetti, M. y Lovreglio, R. 2006. Assessing long-term fire risk at local scale by means of decision tree technique. Journal of Geophysical Research: Biogeosciences, 111(4), 1-15. DoI: 10.1029/2005JG000133.

Aponte, F. 2016. Accesibilidad de los organismos de socorro en la ciudad de Santiago de Cali y su impacto en la población (Tesis de maestría). Universidad Pedagógica y Tecnológica de Colombia. Disponible en: https://ciaf.igac.gov.co/ sites/ciaf.igac.gov.co/files/files_ciaf/UPTC2017-01.pdf.

Armenteras, D., González, F. y Franco, C. 2009. Distribución geográfica y temporal de incendios en Colombia utilizando datos de anomalías térmicas. Caldasia, 31(2), 303-318. Disponible en: https://revistas.unal.edu.co/ index.php/cal/article/view/36107 (Accedido: $15 \mathrm{de}$ septiembre de 2019).

Arpaci, A., Malowerschnig, B., Sass, O. y Vacik, H. 2014. Using multi variate data mining techniques for estimating fire susceptibility of tyrolean forests. Applied Geography, 258-270. Elsevier Ltd. Dor: 10.1016/j.apgeog.2014.05.015.

Bar Massada, A., Syphard, A., Stewart, S. y Radeloff, V. (2012). Wildfire ignition-distribution modelling: a comparative study in the Huron-Manistee National Forest, Michigan, USA. International Journal of Wildland Fire, 22(2), 174-183. DoI: 10.1071/WF11178.

Bisquert, M., Caselles, E., Sánchez, J. y Caselles, V. 2012. Application of artificial neural networks and logistic regression to the prediction of forest fire danger in Galicia using MODIs data. International Journal of Wildland Fire, 21(8), 1025-1029. DoI: 10.1071/WF11105.

Chen, C., Karpatne, A., Chamber, Y., Mithal, V., Lau, M., Steinhaeuser, K., Boriah, S., Steinbach, M. y Kumar, V. 2012. A mew data mining framework for forest fire mapping, in Conference on Intelligent Data Understanding, pp. 771-794. DoI: 10.4018/978-1-5225-5191-1.ch033.

Chen, F., Du, Y., Niu, S. y Zhao, J. 2015. Modeling forest lightning fire occurrence in the Daxinganling mountains of northeastern China with MAXENT. Forests, 6(5), 14221438. DOI: $10.3390 /$ f6051422.

Chuvieco, E., Aguado, I., Yerba, M., Nieto, H., Martin, P., Vilar, L., Martínez, J., Padrón, D. Martín, S. y Salas, J. 2007. Generación de un modelo de peligro de incendios forestales mediante teledetección y sig. Teledetección: hacia un mejor entendimiento de la dinámica global y regional, 19-26.

Correia, M. 2019. Criterios confiables para la construcción del modelo de máxima entropía-MAXENT (Parte 2), 20 de enero, 8-13. Dor: 10.13140/RG.2.2.36673.81769.

Crimmins, M. A. 2006. Synoptic climatology of extreme fire-weather conditions across the southwest United States. International Journal of Climatology, 26(8), 10011016. DoI: $10.1002 /$ joc. 1300.

Díaz-Hormazábal, I. y González, M. E. 2016. Análisis espacio-temporal de incendios forestales en la región del Maule, Chile. Bosque, 37(1), 147-158. Dor: 10.4067/ S0717-92002016000100014.

Díaz, D. 2018. Medición de accesibilidad geográfica entre centros poblados del cantón Tena, Ecuador (Tesis de más- ter). Universidad de Salzburgo. Disponible en: https:// issuu.com/unigis_latina/docs/diaz_checa (Accedido: 20 de octubre de 2019).

Elith, J., Graham, C., Anderson, R., Dudík, M., Ferrier, S., Guisan, A., Hijmans, R., Huettmann, F., Leathwick, J., Lehmann, A., Li, J., Lohmann, L., Loiselle, B., Manion, G., Moritz, C., Nakamura, M., Nakazawa, Y., McC. Overton, J., Townsend, A., Phillips, S., Richardson, K., Scachetti-Pereira, R., Soberón, J., Williams, S., Wisz, M. y Zimmermann, N. (2006). Novel methods improve prediction of species' distributions from occurrence data. Ecography, 29(2), 129-151. Disponible en: http:// rob.schapire.net/papers/nceas.pdf (Accedido: 4 de agosto de 2019).

Eskandari, S. y Chuvieco, E. 2015. Fire danger assessment in Iran based on geospatial information. International Journal of Applied Earth Observation and Geoinformation, 42, 57-64. Elsevier B. v. DoI: 10.1016/j.jag.2015.05.006.

Fick, S. y Hijmans, R. 2017. WorldClim: global climate data. Disponible en: http://worldclim.org/version2 (Accedido: 15 de julio de 2019).

Fielding, A. H. y Bell, J. F. 1997. A review of methods for the assessment of prediction errors in conservation presence/absence models. Environmental Conservation, 24(1), 38-49. DOI: 10.1017/S0376892997000088.

Food and Agriculture Organization of the United Nations. 2000. Communities in flames: Proceedings of an international conference on community involvement in fire management. Editado por Peter Moore et al., Bangkok, Thailand: Regional Office for Asia and the Pacific. Fire Fight South East Asia. Rap Publications. Disponible en: http://www.fao.org/3/AC798E/AC798E00.htm (Accedido: 1 de agosto de 2020).

Galindo, G., Sarango, J. y Villarroel, V. 2012. Los incendios forestales en el Distrito Metropolitano de Quito. Evaluación 2012. Quito. Disponible en: http://www.quitoambiente. gob.ec/ambiente/phocadownload/patrimonio_natural/ Evaluación de los incendios forestales en el 2012.pdf (Accedido: 19 de noviembre de 2018).

Garrido, J., Vilar, L., Echavarría, P., Martínez-Vega, J. y Martín, M. 2018. ¿Pueden las interfaces de usos del suelo explicar la ocurrencia de incendios forestales a escala provincial? Los casos de Zamora y Madrid. Geofocus: Revista Internacional de Ciencia y Tecnología de la Información Geográfica, 22, 71-95. DOI: http://dx.doi. org/10.21138/GF.611.

Ghomi, A., Farahi, E., Baniasadi, R. y Masoumpoor, F. 1937. Rating and mapping fire hazard in the hardwood Hyrcanian forests using GIS and expert choice software, acknowledgement to reviewers of the manuscripts submitted to Forestry Ideas, 19(2), 141-150.

Giglio, L., Boschetti, L., Roy, D., Humber, M. y Justice, C. 2018. Remote sensing of environment the Collection 6 MODIs burned area mapping algorithm and product. Remote Sensing of Environment, (217), 72-85, july. Elsevier. DOI: 10.1016/j.rse.2018.08.005.

Gobierno Autónomo Descentralizado-Municipio de Loja. 2014. Plan de desarrollo y ordenamiento territorial. Loja. 
Gobierno Provincial de Loja. 2016. Guía turística de la provincia de Loja. Prefectura. Editado por D. Cueva. Loja. Disponible en: http://intranet.prefecturaloja.gob. ec/wp-content/uploads/2018/01/turismo.pdf.

Hantson, S., Pueyo, S. y Chuvieco, E. 2014. Global fire size distribution is driven by human impact and climate. Global Ecology and Biogeography, 24(1), 77-86. DoI: 10.1111/geb.12246.

Hong, H., Tsangaratos, P., Ilia, I., Liu, J., Zhu, A. y Xu, C. 2018. Applying genetic algorithms to set the optimal combination of forest fire related variables and model forest fire susceptibility based on data mining models. The case of Dayu county, China. Science of the Total Environment, 630, 1044-1056. Elsevier B. V. DoI: 10.1016/j. scitotenv.2018.02.278.

Huesca, M., González-Alonso, F. y Cuevas, J. 2009. Distribución espacial y temporal de los incendios forestales en España utilizando datos de anomalías térmicas. En Salomón Montesinos Aranda y Lara Fernández Fornos (eds.), Teledetección: Agua y desarrollo sostenible. XIII Congreso de la Asociación Española de Teledetección. Calatayud: 23-26 de septiembre, pp. 309-312. Disponible en: http://www.aet.org.es/congresos/xiii/cal78.pdf (Accedido: 15 de septiembre de 2019).

Ibarra-Montoya, J. y Huerta-Martínez, F. 2016. Modelado espacial de incendios: una herramienta predictiva para el bosque La Primavera, Jalisco México. Revista Ambiente \& Agua, 11(1), 35-49. Dor: 10.4136/1980-993X.

Instituto Nacional de Meteorología e Hidrología. 2018. Publicaciones meteorológicas. Disponible en: http://www. serviciometeorologico.gob.ec/biblioteca/ (Accedido: 5 de agosto de 2019).

Jaiswal, R., Mukherjee, S., Raju, K. y Saxena, R. 2002. Forest fire risk zone mapping from satellite imagery and GIS. International Journal of Applied Earth Observation and Geoinformation, 4(1), 1-10. DOI: 10.1016/S03032434(02)00006-5.

Jin, S. y Sader, S. A. 2005. Modis time-series imagery for forest disturbance detection and quantification of patch size effects. Remote Sensing of Environment, 99(4), 462470. DoI: 10.1016/j.rse.2005.09.017.

Justice, C., Giglio, L., Korontzi, S., Owens, J., Morisette, J. y Roy, D. 2002. The Modis fire products, 83, 244-262. DoI: doi.org/10.1016/S0034-4257(02)00076-7.

Liu, N., Huang, B. y Chandramouli, M. 2006. Optimal siting of fire stations using GIS and ANT algorithm. Journal of Computing in Civil Engineering, 20(5), 361-369. DoI: 10.1061/(ASCE)0887-3801(2006)20:5(361).

Merino-de-Miguel, S., Gonza, F. y Huesca, M. 2011. MoDIs reflectance and active fire data for burn mapping in Colombia. Earth Interactions, 15(10), 17. DoI: 10.1175/2010EI344.1.

National Fire Protection Association. 2018. NFPA 1. Disponible en: https://www.nfpa.org/ (Accedido: 5 de noviembre de 2019).

Ngoc Thach, N., Bao-Toan Ngo, D., Xuan-Canh, P., HongThi, N., Hang Thi, B., Nhat-Duc, H. y Dieu, T. 2018. Spatial pattern assessment of tropical forest fire danger at Thuan Chau area (Vietnam) using GIs-based advanced machine learning algorithms: a comparative study. Ecological Informatics, 46, 74-85. Elsevier B.v. DoI: 10.1016/j.ecoinf.2018.05.009.

Ocles, M. A. 2008. Red Interamericana de Mitigación de Desastres. Secretaría de Gestión de Riesgos. Disponible en: http://www.rimd.org/organizacion.php?id=189 (Accedido: 16 de noviembre de 2019).

Oliveira, S., Oehler, F., San-Miguel-Ayanz, J., Camia, A. y Pereira, José M. 2012. Modeling spatial patterns of fire occurrence in Mediterranean Europe using multiple regression and random forest. Forest Ecology and $M a-$ nagement, 275, 117-129. Elsevier B. v. DOI: 10.1016/j. foreco.2012.03.003.

Parisien, M., Snetsinger, S., Greenberg, J., Nelson, C., Schoennagel, T., Dobrowski, S. y Moritz, M. 2012. Spatial variability in wildfire probability across the western United States. International Journal of Wildland Fire, 21(4), 313-327. Dor: 10.1071/WF11044.

Parolo, G., Rossi, G. y Ferrarini, A. (2008). Toward improved species niche modelling: Arnica montana in the Alps as a case study. Journal of Applied Ecology, 45(5), 1410-1418. DOI: 10.1111/j.1365-2664.2008.01516.x.

Peterson, A., Egbert, S., Sánchez-Cordero, V. y Price, K. (2000). Geographic analysis of conservation priority: endemic birds and mammals in Veracruz, México. Biological Conservation, 93(1), 85-94. DoI: 10.1016/ S0006-3207(99)00074-9.

Phillips, S. J., Anderson, R. P. y Schapire, R. E. (2006). Maximum entropy modeling of species geographic distributions. Ecological Modelling, 190(3-4), 231-259. Elsevier. DOI: 10.1016/J.ECOLMODEL.2005.03.026.

Pourghasemi, H. R. (2016). GIs-based forest fire susceptibility mapping in Iran: a comparison between evidential belief function and binary logistic regression models. Scandinavian Journal of Forest Research, 31(1), 80-98. DOI: 10.1080/02827581.2015.1052750.

Renard, Q., Plissier, R., Ramesh, B. y Kodandapani, N. (2012). Environmental susceptibility model for predicting forest fire occurrence in the Western Ghats of India. International Journal of Wildland Fire, 21(4), 368-379. DoI: 10.1071/WF10109.

Rodríguez Montellano, A. 2012. Cartografía multitemporal de quemas e incendios forestales en Bolivia: detección y validación post-incendio. Ecología en Bolivia, 47(1), 53-71. Disponible en: https://url2.cl/9mvCr (Accedido: 12 de noviembre de 2019).

Roteta, E., Bastarrika, A., Padilla, M., Storm, T. y Chuvieco, E. 2019. Development of a Sentinel-2 burned area algorithm: generation of a small fire database for sub-saharan Africa. Remote Sensing of Environment, 222, 1-17. Elsevier Inc. DoI: 10.1016/j.rse.2018.12.011.

Running, S. W. 2006. Is global warming causing more, larger wildfires? Science, 313(5789), 927-928. DOI: 10.1126/ science. 1130370.

Sachdeva, S., Bhatia, T. y Verma, A. K. 2018. GIs-based evolutionary optimized gradient boosted decision trees for forest fire susceptibility mapping. Natural Hazards, 
92(3), 1399-1418. Springer Netherlands. DoI: 10.1007/ s11069-018-3256-5.

Satchet, M. S., Muhammad-Ali, A. H., Khalaf, Y. H., Jaber, A. S. y Wanas, S. K. 2018. Assessment and development of the spatial coverage of fire service in Nasiriyah city by using geographic information systems (GIS). International Journal of Civil Engineering and Technology (IJCIET), 9(7), 936-947. Disponible en: https://url2.cl/ ELtz4 (Accedido: 18 de noviembre de 2019).

Savsar, M. 2014. Fire station location analysis in a metropolitan area. International Journal of Industrial and Systems Engineering, 16(3), 365-381. Inderscience Enterprises Ltd. DoI: 10.1504/IJISE.2014.060134.

Secretaría Nacional de Gestión de Riesgos y Emergencias. 2017. Propuesta metodológica. Susceptibilidad a incendios forestales. Quito.

Secretaría Nacional de Gestión de Riesgos y Emergencias. 2016. Guía operativa para la organización y funcionamiento de los cuerpos de bomberos a nivel nacional. Samborondón. Disponible en: https://url2.cl/NSkwi (Accedido: 13 de noviembre de 2018).

Soto, F. 2017. Cartografía, evaluación y seguimiento de incendios forestales de la zona sur del Ecuador usando datos satelitales (Tesis de pregrado). Universidad Técnica Particular de Loja.

Stockwell, D. R. B. y Peterson, A. T. 2002. Effects of sample size on accuracy of species distribution models. Ecological Modelling, 148(1), 1-13. DoI: 10.1016/S03043800(01)00388-X.

Sturtevant, B. R. y Cleland, D. T. 2007. Human and biophysical factors influencing modern fire disturbance in northern Wisconsin. International Journal of Wildland Fire, 16(4), 398-413. DoI: 10.1071/WF06023.
Syphard, A., Radeloff, V., Keeley, J., Hawbaker, T., Clayton, M., Stewart, S. y Hammer, R. 2007. Human influence on California fire regimes. Ecological Applications, 17(5), 1388-1402. DOI: 10.1890/06-1128.1.

Tien Bui, D., Bui, Q., Nguyen, Q., Pradhan, B., Nampak, H. y Trinh, P. 2017. A hybrid artificial intelligence approach using GIs-based neural-fuzzy inference system and particle swarm optimization for forest fire susceptibility modeling at a tropical área. Agricultural and Forest Meteorology, 233, 32-44. Elsevier B.v. DoI: 10.1016/j. agrformet.2016.11.002.

Vilar del Hoyo, L., Gómez Nieto, I., Martín Isabel, M. y Martínez Vega, F. 2007. Análisis comparativo de diferentes métodos para la obtención de modelos de riesgo humano de incendios forestales. Proceeding of IV International Wildfire Conference, 13-17. Disponible en: https://url2. cl/35X91 (Accedido: 20 de octubre de 2019).

Welsh, A., Cunningham, R., Donnelly, C. y Lindenmayer, D. 1996. Modelling the abundance of rare species: statistical models for counts with extra zeros. Ecological Modelling, 88(1-3), 297-308. DOI: 10.1016/0304-3800(95)00113-1.

Zhang, G., Wang, M. y Liu, K. 2019. Forest fire susceptibility modeling using a convolutional neural network for Yunnan province of China. International Journal of Disaster Risk Science. Beijing Normal University Press, 10(3), 386-403. DOI: 10.1007/s13753-019-00233-1.

Zhang, W. y Jiang, J. 2012. Research on the location of fire station based on GIS and GA. Applied Mechanics and Materials, (130-134), 377-380. DoI: 10.4028/www. scientific.net/AMM.130-134.377. 